\title{
Phase space structures generated by absorbing obstacles in streaming plasmas
}

\author{
P. Guio ${ }^{1}$ and H. L. Pécseli ${ }^{2,3}$ \\ ${ }^{1}$ Parallab, Bergen Center for Computational Science, Thormøhlensgate 55, N-5008 Bergen, Norway \\ ${ }^{2}$ University of Oslo, Physics Department, Blindern, N-0316 Oslo, Norway \\ ${ }^{3}$ Centre for Advanced Study, Drammensveien 78, N-0271 Oslo, Norway
}

Received: 30 September 2004 - Revised: 19 November 2004 - Accepted: 15 December 2004 - Published: 30 March 2005

\begin{abstract}
The dynamic behavior of a collisionless plasma flowing around an obstacle is investigated by numerical methods. In the present studies, the obstacle is formed by an absorbing cylinder, and a 2-D electrostatic particle-in-cell simulation is used to study the flow characteristics, with extensions to a fully 3-D generalization of the problem demonstrated as well. The formation of irregular filamented density depletions, oblique to the flow, is observed. The structures form behind the obstacle, in a region with a strong velocity shear, but also other instability mechanisms can be identified. The dynamics of these structures is highly dependent on the physical parameters of the plasma, and they can either be quasi-stationary or undergo a dynamic evolution. The structures are found to be associated with phase-space vortices, observed especially in the phase space spanned by the velocity direction perpendicular to the flow and the spatial coordinate in the same direction. The bias of the obstacle with respect to the plasma potential is found to be an important parameter for the dynamics of the structures, but seemingly not for their formation as such. The results can be of interest in the interpretation of structures in space plasmas as observed by instrumented spacecrafts.
\end{abstract}

Keywords. Space plasma physics (Numerical simulation studies; Electrostatic structures; Spacecraft sheaths, wakes, charging)

\section{Introduction}

The properties and dynamics of collisionless plasmas can be strongly influenced by inhomogeneities and boundaries, and such conditions have been studied in some detail, with results reported in the literature. For instance, the problem of plasma flow past an obstacle has been studied for different system sizes, as well as for different plasma parameter regimes. For example, Stangeby and Allen $(1970,1971)$ studied analytically the stationary flow pattern of a compress-

Correspondence to: $\mathrm{H}$. L. Pécseli

(hans.pecseli@fys.uio.no) ible, supersonic, irrotational and inviscid flow of drifting cold ions around a cylindrical obstacle. These results are of interest for the interpretation of probe characteristics, but also in other contexts, such as dust particles (Shukla, 2002) in a gas discharge or dust-grains in plasmas occurring in nature, where the rings of Saturn represent such an example (Hartquist et al., 2003). Considering different and much larger length scales, Farrell et al. (1997, 1998); Birch and Chapman $(2001,2002)$ studied the kinetic effects of solar wind electrons and the interplanetary magnetic field (IMF) direction in the wake formation and evolution past the Moon.

In the present study, we consider small absorbing cylindrical obstacles, with diameters on the Debye length scale or larger, immersed in a plasma consisting of an ion species having a net drift velocity relative to the obstacle (Guio and Pécseli, 2004). We investigate the characteristics of fluctuations excited behind the obstacle under these conditions. The problem is analyzed by numerical methods, using a particlein-cell (PIC) code. The interesting observation here is not only that fluctuations develop in the wake behind the obstacle, but that they can take the form of long-lived structures, which at closer inspection turn out to be associated with phase space vortices, which are known from laboratory experiments, as well as several numerical plasma simulations. The analysis can have implications for the study of plasma waves detected by instrumented spacecraft, where it seems that care should be taken to distinguish between fluctuations generated by the spacecraft from those naturally occurring in the ambient plasma.

The geometry of the problem posed here resembles that of low Mach number neutral winds flowing past an obstacle, a smokestack, for instance. This results in the formation of the well-known "von Karman vortex street". In that problem (in its standard form), however, the flow is treated as incompressible. For the present related plasma counterpart of the problem, the compressibility of the medium is crucial. In neutral fluids, momentum is imparted to neighboring fluid elements by pressure and viscosity, in collisionless plasmas with conditions given in the present study, by electrostatic fields. 
The present paper is organized as follows: in Sect. 2 we give an outline of the numerical methods, with results presented in Sect. 3. In Sect. 4 we discuss possible instability mechanisms active for generating the observed structures, and identify a velocity shear, as well as an ion-ion two-stream instability, where one or both may be active for various parameter regimes. Finally, Sect. 5 contains our discussions, including, in particular, a discussion of the relevance for some spacecraft observations.

\section{Numerical simulations}

A general particle simulation describes both ions and electrons as discrete particles, which in a purely electrostatic model are propagated by the collective electric fields. These models are numerically very time consuming because of the significant difference in the characteristic time scales of ions and electrons (i.e. the relevant plasma periods). An often assumed simplification consists of retaining a particle model for the ion dynamics, but simplify the treatment of the electrons by assuming a fluid model for this component. This approximation is generally assumed to be justified for phenomena on time scales longer than the ion plasma period. In the present study, we use the same particle-in-cell simulator as Guio et al. (2001, 2003); Guio and Pécseli (2003), with the only extension that it allows for inclusion of absorbing surfaces. The code is parallelized and allows for simulations with a large number of particles over a wide rectangular region. The electrons are assumed to be thermalized at temperature $T_{e}$, and to be Boltzmann distributed at all times, leading to the nonlinear Poisson equation

$\nabla^{2} \phi=\frac{e}{\epsilon_{0}}\left(n_{0} \exp \left(\frac{e \phi}{T_{e}}\right)-n\right)$,

where $n$ is the ion density, assuming singly charged ions. Quasi-neutrality is thus not explicitly assumed. Under some special conditions, with additional assumptions, we can generalize the model for an electron Boltzmann distribution slightly (Guio et al., 2003).

Because of the assumed isothermal Boltzmann distribution of the electron component, our code does not account for electron kinetic phenomena due to, for instance, distorted electron velocity distribution functions, or electron current instabilities. The model can be justified for cases where the potential difference between the obstacle and the plasma is small, and in particular cases where the electron-electron collisions are significant, while the ion component can be considered collisionless. The code offers a great advantage in needing attention only to the time scale of the ion dynamics. Since electron inertia is ignored in the present approximation, a slow electron flow is irrelevant for the analysis. Larger electron flows will require a more time consuming code, where the electrons are also treated as individual particles. The present code allows for an externally applied magnetic field (Guio et al., 2001, 2003; Guio and Pécseli,
2003), but for the present results we consider an unmagnetized plasma. Phase space structures can be formed in magnetized plasma as well (Børve et al., 2001; Daldorff et al., 2001; Guio et al., 2003; Jovanović and Shukla, 2003).

The problem is analyzed by a nonlinear multi-grid method which consists of a full multi-grid scheme, with an adaptive strategy that allows for the omission of a coarse-grid correction if the specified accuracy is already reached on that grid. This scheme might be followed by some iterations of adaptive multi-grid schemes, in order to improve the accuracy (Wesseling, 1991). The relaxation method uses a GaussSeidel-Newton iteration scheme. The simulation region here is $120 \lambda_{i} \times 120 \lambda_{i}$, in units of the ion Debye length $\lambda_{i}$. We have typically $3 \times 10^{6}$ particles in a simulation. The analysis allows for the inclusion of multi-electron temperature distributions (Guio et al., 2003), but these were not studied in any systematic way here.

An absorbing circular obstacle is placed at the origin of the simulation region. Every ion crossing the surface of the obstacle is removed from the simulation, and the net charge density inside the object is fixed to zero. This solution allows for a simple treatment of the obstacle, which is here assumed to be at a constant potential, equal to the plasma potential. First, a $10 \lambda_{i}$ diameter is considered, but also conditions with other diameters have been analyzed. The object is of course subject to a drag force (Ivlev et al., 2004), which is, however, ignored here. This is justified if the inertia of the object is large, as assumed here, and the drag will then not be manifested for the durations of the simulations presented in this analysis.

The ions have a net flow along the $y$-direction, as ensured by injection of particles at one boundary. The boundary condition applied to the particles along the flow as well as the perpendicular direction, is a free space boundary, with the number of injected particles being calculated from the flux of a Maxwellian distribution. The boundary condition for the electric field is a Neumann condition along the flow direction, and a Dirichlet condition in the perpendicular direction. In this way, the quasi-neutrality condition at the boundary is fulfilled. Some preliminary results of the present studies were published previously (Guio and Pécseli, 2004).

\section{Numerical results}

One of the most important parameters of the problem is the velocity difference between the plasma and the obstacle, i.e. the Mach number associated with the problem. If we choose the ion flow velocity to be supersonic, we obtain the wellknown sound-wave cones, very similar to related previous results (Guio and Pécseli, 2003), where we studied radiation of sound from a supersonic point charge. One interesting feature of the present analysis is that only moderate flow velocities are used, well below $C_{s}$, but larger than the ion thermal velocity. In the following we measure time in units of $\Omega_{p i}^{-1}$, where $\Omega_{p i}$ is the ion plasma period, spatial separations in units of the ion Debye length $\lambda_{i}$, and velocities in terms 

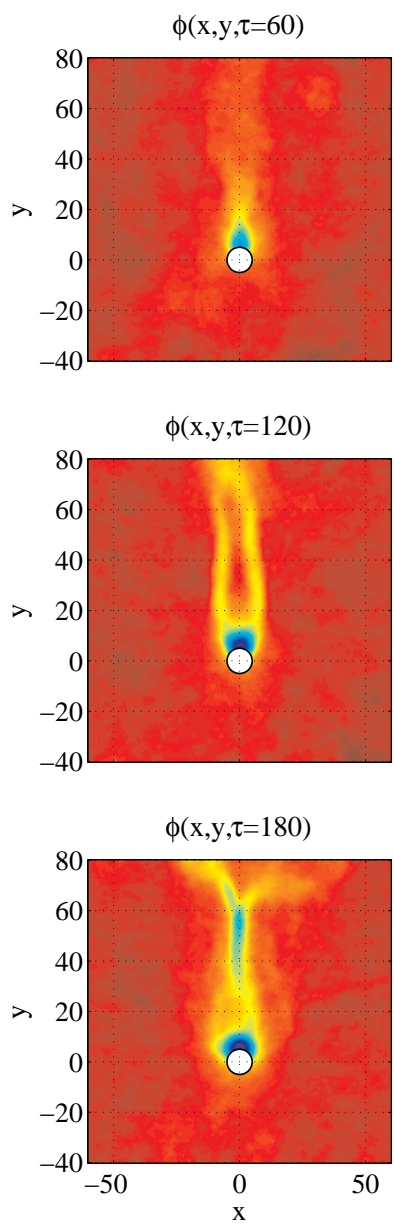
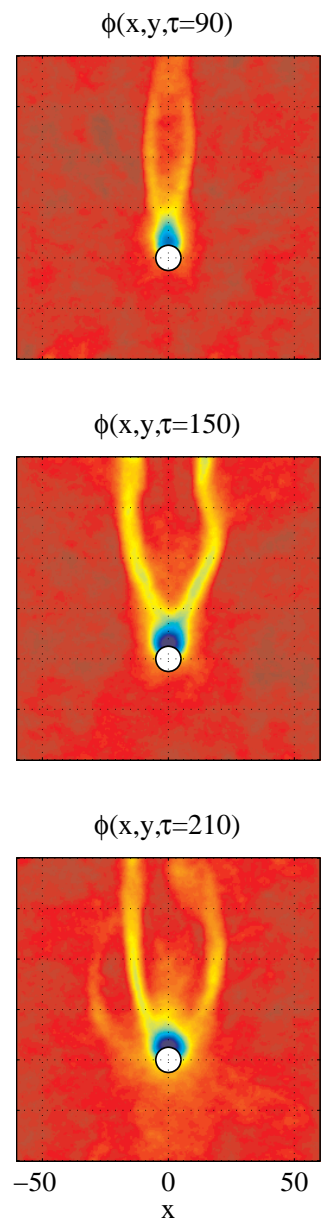

Fig. 1. Two-dimensional electrostatic potential at 6 different times of the simulation. The color scale has red as maximum, where $e \phi / T_{i} \approx 0.2$ and deep blue as minimum, where $e \phi / T_{i} \approx-2.5$, slightly behind the obstacle. The ion drift velocity is $V_{0}=0.22 \times C_{S}$.

of $\sqrt{T_{i} / M}$, while potentials are normalized by $T_{i} / e$, measuring temperatures in energy units, thus omitting Boltzmann's constant.

Figure 1 shows the two-dimensional electrostatic potential at 6 different times. For this particular simulation we have $T_{e} / T_{i 0}=20$, but the results are representative for other smaller temperature ratios as well. To simulate conditions in nature, where the ion temperature is often largest in the direction of the ion flow, we used $T_{i \|} \equiv T_{i 0}=5 T_{i \perp}$ for the ion population injected at the boundary. It turns out, however, that neither this temperature anisotropy seems to be of any major importance.

We note first the formation of a density depletion behind the obstacle, with an associated potential variation given by Eq. (1). The density depletion breaks-up into long filaments behind the obstacle. In order to visualize the time variation of the potential associated with the filaments we place a dense probe array positioned in the $x$-direction (perpendicular to the flow velocity), at a downstream position of $40 \lambda_{i}$. The time evolution is sampled for 400 ion plasma periods.

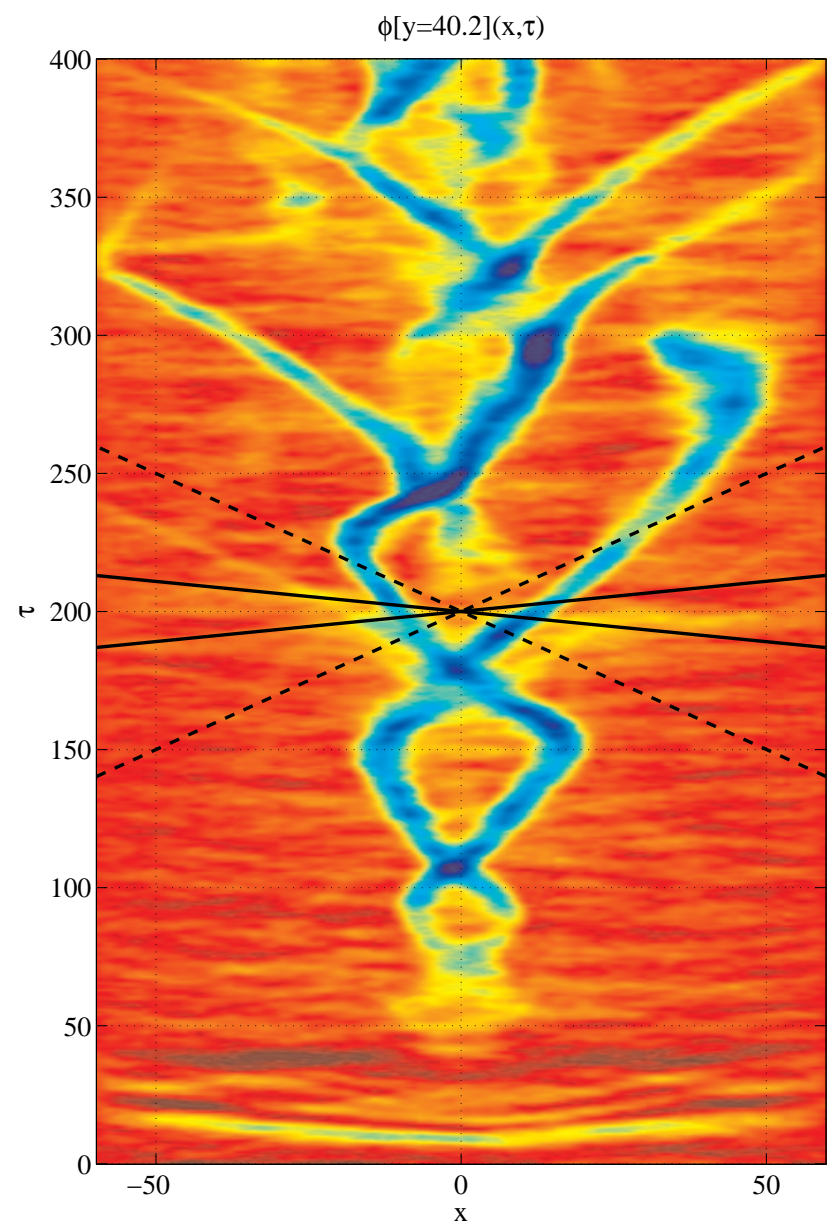

Fig. 2. Time variation of the electric potential detected at a probe array placed at a downstream position of $40 \lambda_{i}$. The time evolution is sampled with the numerical time resolution for 400 -ion plasma periods, for parameters as in Fig. 1. As a reference, we inserted the slopes of the ion sound velocity (solid line) and the ion thermal velocity (dashed line), in the middle of the figure. The color scale has red as maximum, where $e \phi / T_{i} \approx 0.2$ and deep blue as minimum, where $e \phi / T_{i} \approx-1.4$.

The widths of the structures after saturation is approximately $10 \lambda_{i}$. Results are shown in Fig. 2. The formation time of the structures is approximately 100 ion plasma periods.

We observe a rather irregular motion of the two filaments, sometimes they cross, and sometimes one may be terminated, to reappear in a different position along the probe array. At several times, we note a coalescence of two structures, e.g. at $t \approx 105$ and $t \approx 180$. The local slope of the structures in the $\{x, t\}$-diagram gives the local velocity component in the $x$ direction, which is here the dominant one. Disruptions, and other abrupt changes of the structures are often associated with radiation of small amplitude slow waves: an example is noticeable, for instance, in Fig. 2 , around $(x, t) \approx(0,180)$. The $x$-component of the velocity of these fluctuations is found to be between the ion thermal velocity and the speed of sound. If we reduce the electron-ion temperature ratio, filaments still form, but they become thinner and more irregular. 

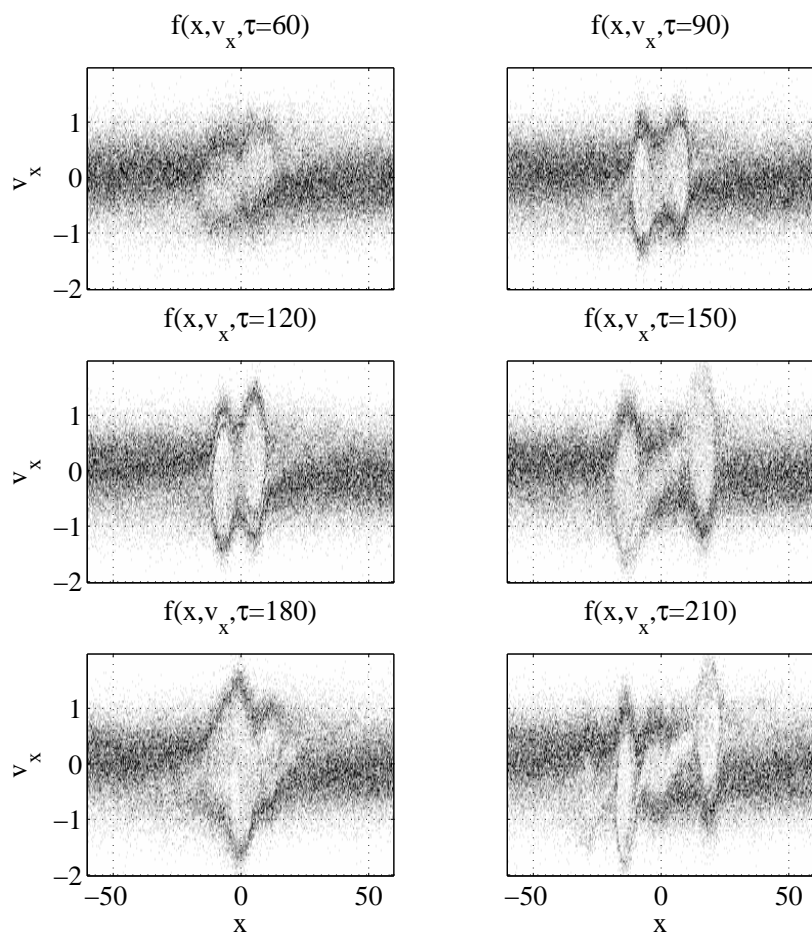

$\mathrm{f}(\mathrm{x}, \mathrm{v}, \tau=150)$

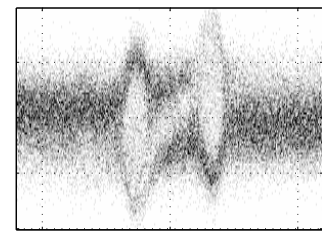

$\mathrm{f}\left(\mathrm{x}, \mathrm{v}_{\mathrm{x}}, \tau=210\right)$

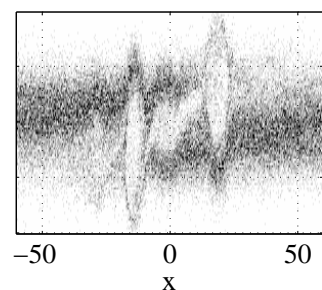

Fig. 3. A section of phase space, spanned by $\left\{x, v_{x}\right\}$, shown at selected times, for parameters as in Fig. 1, and the same $y$-position as in Fig. 2.

While the present parameters typically give two filaments, as in Fig. 1, a reduced temperature ratio can give a larger number. In all cases they start out from the wake or shadow region immediately behind the obstacle.

The presentations in Fig. 2 are in terms of plasma potential, but since the structures are larger than the Debye length, the quasi-neutrality condition is approximately fulfilled, and the figures are representative for the variations in plasma density as well.

Based on the potential variation alone, it is not possible to make any further definite statements concerning the nature of the observed structures. In order to obtain a better understanding, we analyze the phase space spanned by the $\left\{x, v_{x}\right\}$-coordinates, at selected times. Results are shown in Fig. 3, and for the $\left\{x, v_{y}\right\}$ part of phase space in Fig. 4. The conspicuous features of the potential structures seem to be associated with phase space vortices, where all the relevant dynamics seem to take place in $\left\{x, v_{x}\right\}$-plane, as expected for potential structures elongated in the $y$-direction, as here. Such ion phase space vortices are well-known, and have been observed experimentally by Pécseli et al. (1981, 1984), as well as in a number of numerical simulations (Sakanaka, 1972; Børve et al., 2001; Daldorff et al., 2001; Guio et al., 2003). In particular, the coalescence seen in Fig. 2 is associated with a phase space coalescence, as found in Fig. 3, at, for instance, $t=180$. The velocities of these structures seems to be at or below the ion thermal velocity, consistent with analytical results, which demonstrate that these Bernstein-
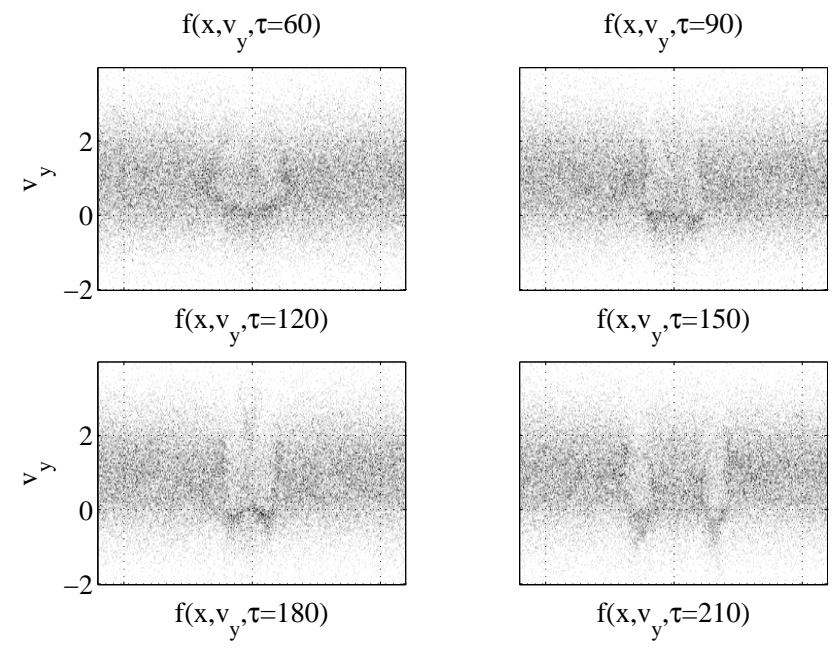

$\mathrm{f}\left(\mathrm{x}, \mathrm{v}_{\mathrm{y}}, \tau=210\right)$
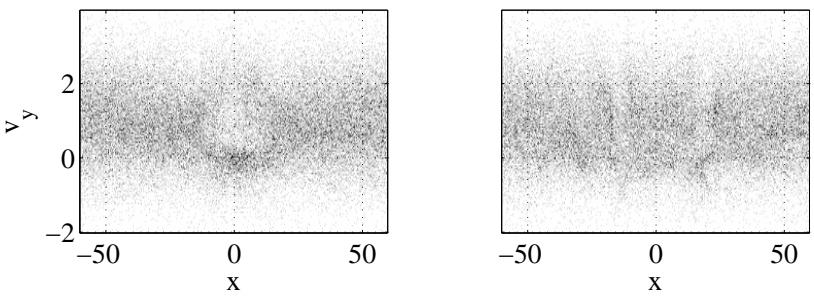

Fig. 4. A section of phase space, spanned by $\left\{x, v_{y}\right\}$, shown at selected times, for parameters as in Fig. 1, and the same $y$-position as in Figs. 2 and 3. Note that the grey-scale has been changed in comparison with Fig. 3, in order to emphasize the weaker phase space structure.

Greene-Kruskal (BGK) equilibria (Bernstein et al., 1957) can have in principle any characteristic velocity, as long as they are embedded into the velocity distribution function. In their analysis of ion phase space structures, or "solitary ion holes", Schamel and Bujarbarua (1980) and Bujarbarua and Schamel (1981) found an upper limit for their velocity to be 1.4-ion thermal speeds. The radiation of small amplitude fluctuations here is seemingly associated with changes in ion phase space. It is interesting to also note that Singh et al. (2001) observed radiation of small amplitude waves when phase space vortices underwent rapid changes (in their case electron holes radiating plasma waves). Related observations were made by Newman et al. (2001) and Oppenheim et al. (2001).

To illustrate the parameter variation, we show, in Fig. 5, the normalized potential variation for the case where the temperature ratio $T_{e} / T_{i}$ is moderate, but still with sub-sonic flow parameters. The corresponding phase space presentation is shown in Fig. 6. We find that in this case it takes longer for the phase space structures to form, and they appear somewhat more "blurred" as compared to the case illustrated by Figs. 1 and 3, but this is to some extent an artifact of the choice of positions. We choose to represent the features in the same spatial positions for the two cases, but if we take a position further downstream, where the structures are more strongly developed, see Fig. 5, then the phase space structures will also be better defined. 

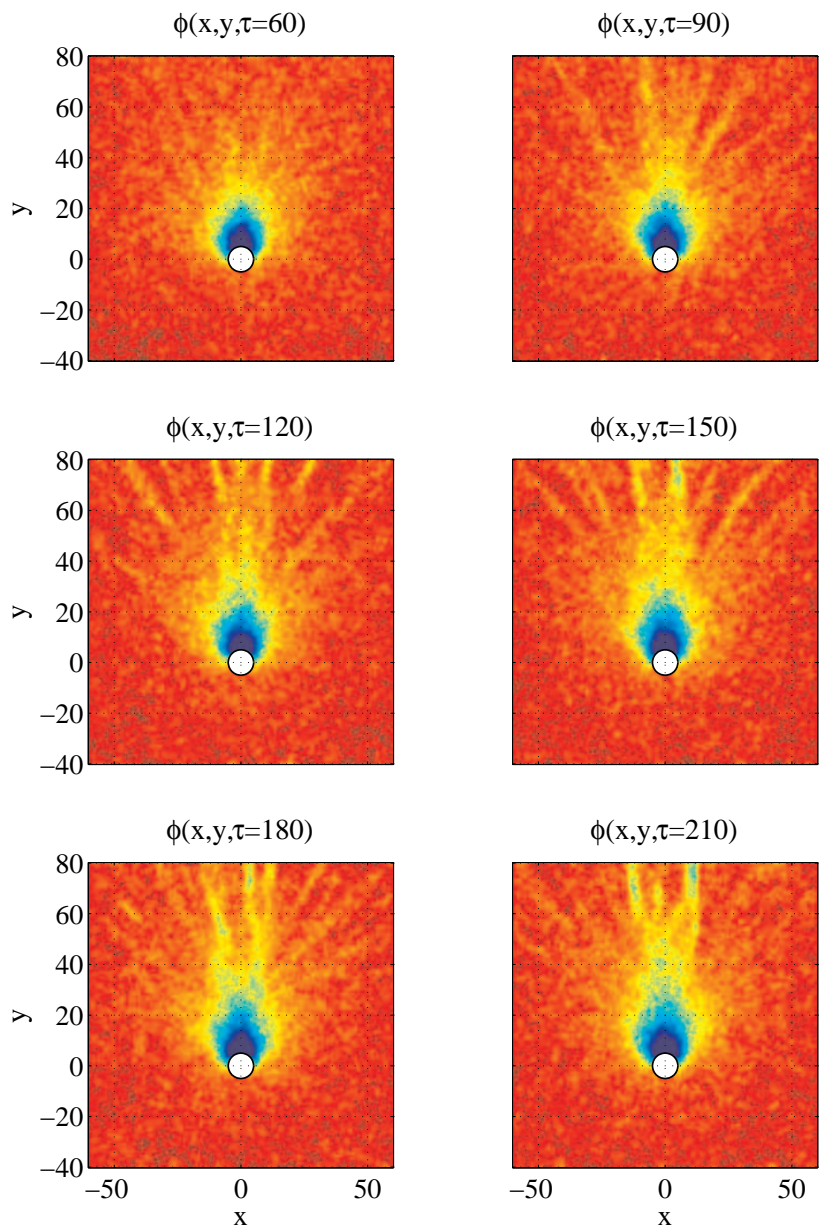

Fig. 5. Two-dimensional electrostatic potential at 6 different times of the simulation. The color scale has red as maximum, where $e \phi / T_{i} \approx 0$ and deep blue as minimum, where $e \phi / T_{i} \approx-0.5$, slightly behind the obstacle. Here we have $T_{e}=2 T_{i}$ for isotropic ion temperatures. The ion drift velocity here is $0.82 \times C_{S}$.

As mentioned, we assumed that the obstacle was at the plasma potential, so the only way it interacts with the ions is by adsorption, corresponding to a recombination of ions at the surface. It does not change the results in any appreciable way to let the obstacle be biased, however. We show in Fig. 7 a case where the bias is $\Psi_{o}=-4 T_{e} / e$, which is approximately the potential we would have in case the obstacle was at floating potential. We see that the potential evolution behind the obstacle is noticeably more irregular, as compared to the case without bias. Assuming a steady state, Tskhakaya et al. (2004) studied the formation of the sheath around a floating object, in their case a dust grain moving with respect to the plasma, and found the sheaths to be deformed due to the flow. We find the sheath to be highly dynamic in our case and observe that phase space structures are forming here as well, see Fig. 6. We find that a larger obstacle (e.g. 20 $\lambda_{i}$ ) basically gives the same irregular features, and argue that the essential effect of a bias on the obstacle is to make its effective cross section larger, due to the surrounding sheath. In
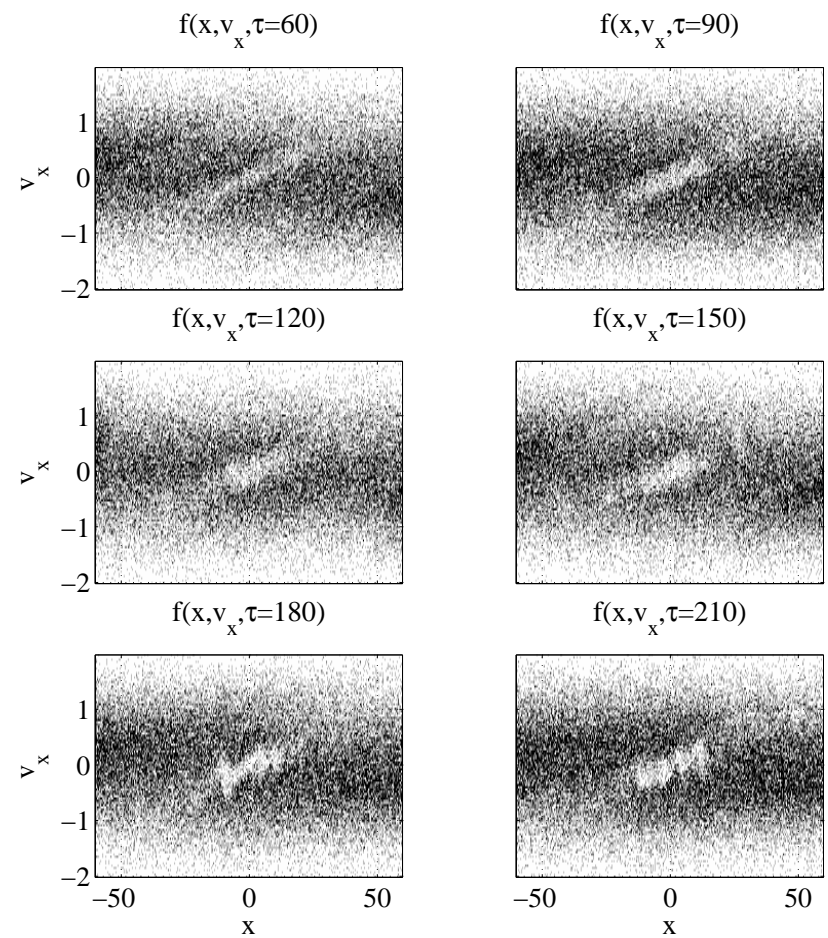

Fig. 6. A section of phase space, spanned by $\left\{x, v_{x}\right\}$, shown at selected times, for parameters as in Fig. 5. Note that the grey-scale has been changed in comparison with Fig. 3, in order to emphasize the weaker phase space structure.

particular, Fig. 7 shows that the structures can appear on virtually any position with respect to the axis of the obstacle, i.e. not necessarily in the wake of the flow.

In Fig. 8 we show a sample of phase space corresponding to the data in Fig. 7. We can clearly identify two "beams" in this phase space, and also the formation of phase space vortices in this case. Note, however, that the vortices form on the "high velocity" sides of the beams (seen best for early times), in contrast to what is expected for vortices formed by kinetic, beam-type, instabilities. We attribute this to be a consequence of the velocity shear, to be discussed later.

We note that an ion flow is known to modify the sheath characteristics (Pal et al., 2002; Kwok et al., 2003). The large-scale motion of the vortices in the present case, where it is significantly more noticeable than when the obstacle is at plasma potential, is due to the fluctuations in the sheath, which act to scatter the phase space structures being formed. In particular, we note that the structures in the plasma seem basically to be constrained to the wake of the solid obstacle when it is biased to the plasma potential. When the object is at, or close to, the floating potential, the structures can at times be found in front of, or up streams of the obstacle.

To substantiate the discussion of the fluctuations of the sheath in Fig. 7, we show in Fig. 9 short samples obtained at 4 positions inside the sheath around the object. First, we note a short transient after the beginning of the simulation, followed by some low frequency oscillations. We note 

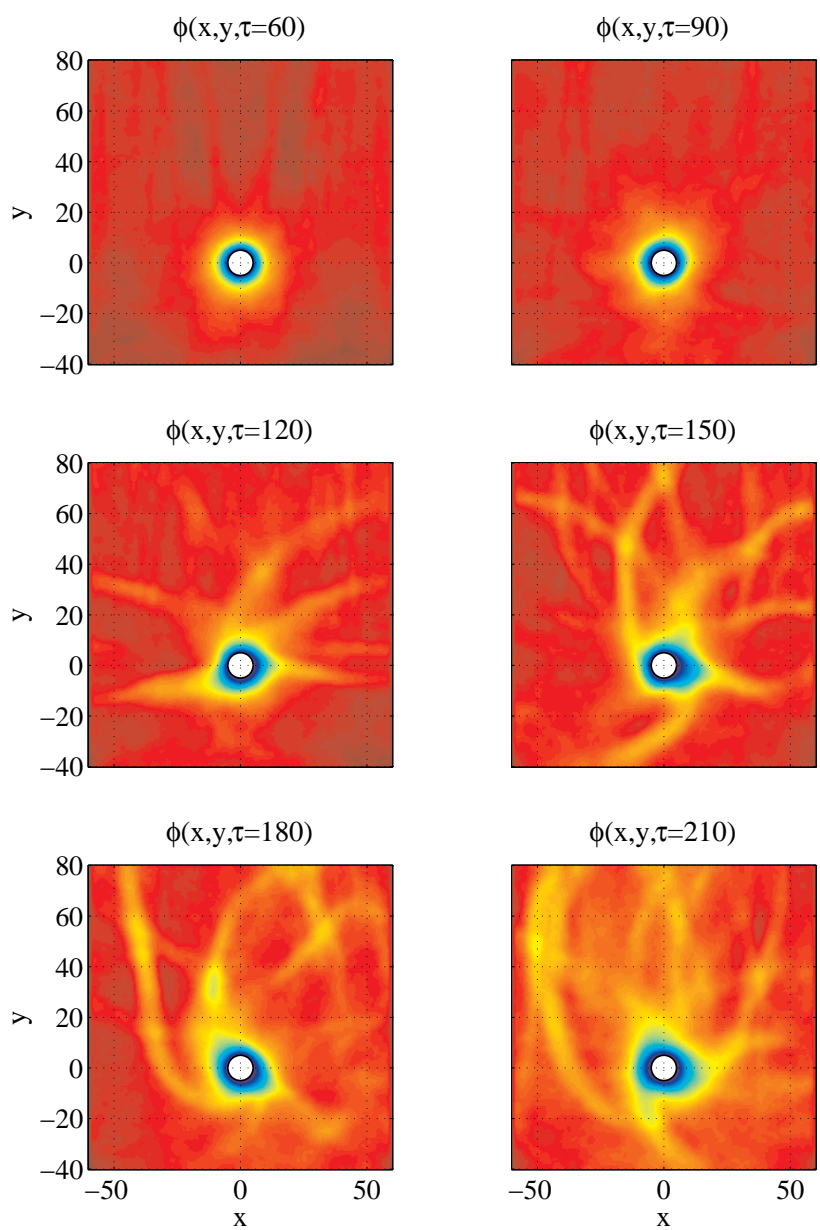

Fig. 7. Two-dimensional electrostatic potential at 6 different times of the simulation. The color scale has red as maximum, where $e \phi / T_{i} \approx 0$ and deep blue as minimum, where $e \phi / T_{i} \approx-6$, slightly behind the obstacle. We have plasma parameters as in Fig. 1, but in the present case the obstacle is at a potential of $-4 T_{e} / e$.

that the oscillations are seemingly in phase around the obstacle, indicating that the dominant oscillation here is a uniform "pulsation" of the sheath. The peak-to-peak amplitudes are sufficient to cause a nontrivial scattering of ions. By considering extended simulation periods, we obtain spectra as those shown in Fig. 10. We note the peaks around 0,05-0.07 $\Omega_{p i}$, and also that there seems to be some variations with positions around the object. This latter observation can in part be due to the finite record length used here, but in principle, we would expect at least some difference between the "up" and "down" positions. We find it interesting that the periods around $15-20 \Omega_{p i}^{-1}$ for the peaks observed in the spectrum are close to $D / V_{0}$. This parameter variation deserves further scrutiny, but that study is outside the scope of the present analysis.

We find it relevant to analyze object potentials, $\Psi_{o}$, at the plasma, as well as the floating potential. In a laboratory, obviously the bias can be externally imposed, but on a spacecraft there are possibilities for at least some controlled
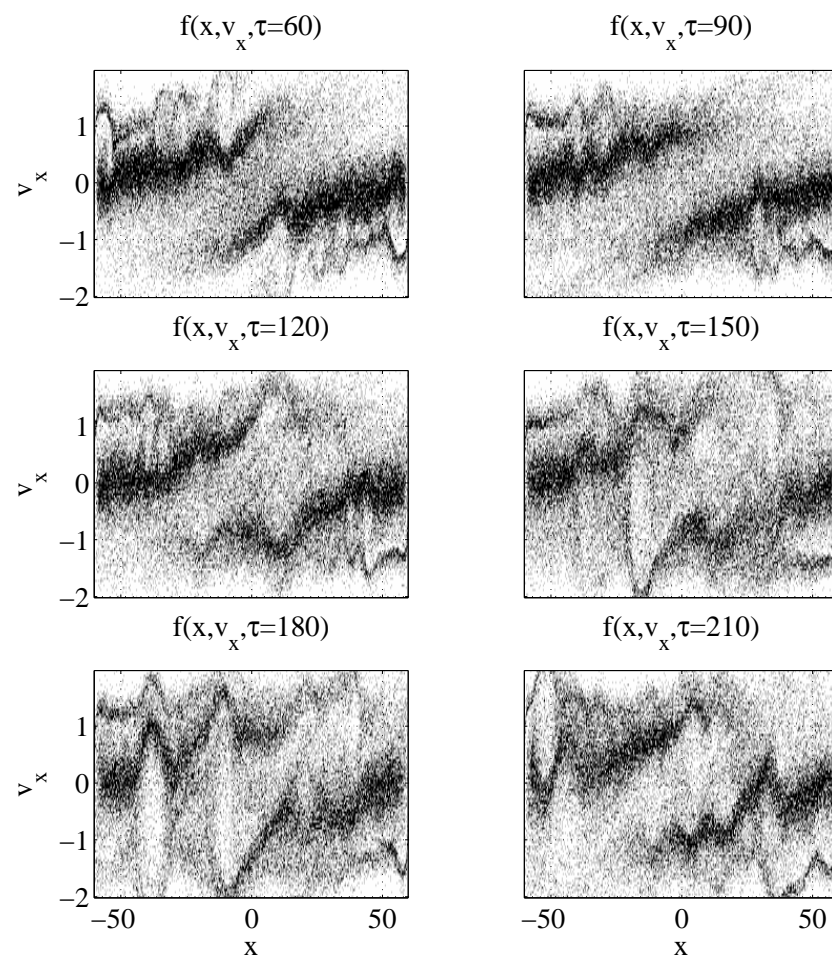

Fig. 8. A section of phase space, spanned by $\left\{x, v_{x}\right\}$, shown at selected times, for parameters as in Fig. 7, and the same $y$-position as in Fig. 2. Note the formation of phase space vortices on the high velocity side of the beams, seen best for early times.

adjustments as well. Actually, in such cases positive potentials, corresponding to electron absorption, are also relevant, but the assumption of Boltzmann distributed electrons inherent in our simulations prohibit us from studying this limit, where the electron current drawn to the solid object can give rise, for instance, to plasma instabilities of a nature different from those studied in the present work.

\subsection{Three spatial dimensions}

We have investigated the present problem by a fully threedimensional code as well. That analysis is significantly more time consuming, but gives essentially the same results. As an illustration, we show in Fig. 11 a three-dimensional presentation of the potential variation, where we have a cylinder segment embedded in the flowing plasma (see also Fig. 12 for the potential variation in a selected plane for 6 different times). The plasma parameters are as in Fig. 1, apart from having $T_{i \|}=T_{i \perp}$ here. Also in this case, we see clearly the formation of spatially extended filaments. Our observations are therefore not restricted by the dimensionality of the code. We note though, that in the simulation from Fig. 11 if we replace the cylinder segment with a sphere having the same diameter as the cylinder, we see a diffuse noise level behind the sphere almost without structures. These will re-occur only if the radius is substantially increased. In order to find structures developing in the plasma flow for reduced temperature 
$\phi$ (left probe)

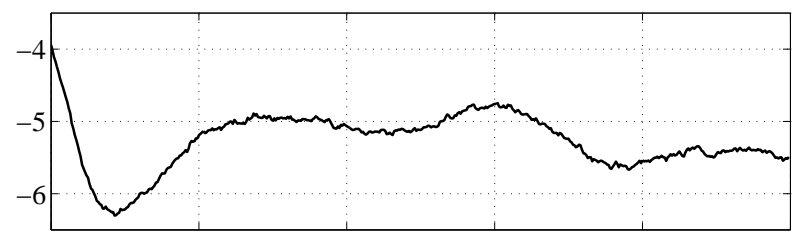

$\phi$ (lower probe)

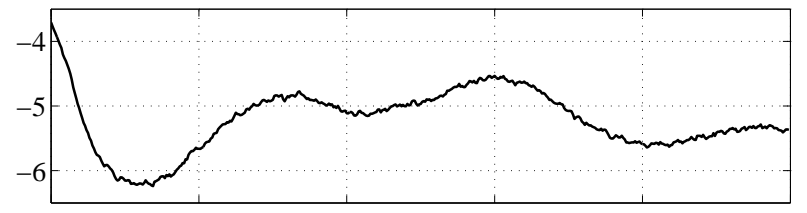

$\phi$ (right probe)

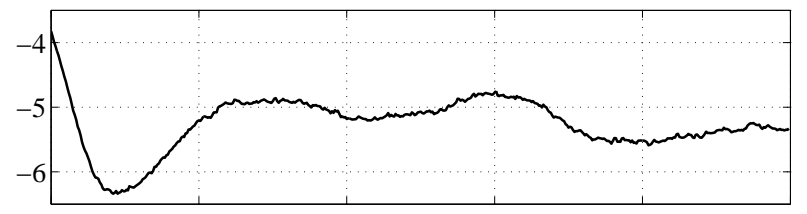

$\phi$ (upper probe)

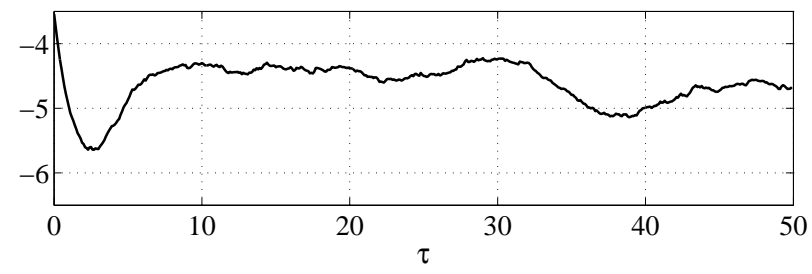

Fig. 9. Potential variation obtained from 4 positions inside the sheath around the object, see Fig. 7. The notation used here refers to the figure, i.e. "upper" means a position in the shadow behind the object, etc. Times here are also in units of $\Omega_{p i}^{-1}$.

ratios, $T_{e} / T_{i}$, it is particularly important to have objects being strongly elongated.

In general, the formation of phase space structures seems to require $T_{e}>T_{i}$, which is in agreement with numerical studies by Pécseli et al. (1981), and has analytical support in the work by Bujarbarua and Schamel (1981). Enhanced electron temperatures are frequent in the Earth's upper ionosphere and magnetosphere. An ion temperature anisotropy seems to be of minor importance, presumably because the ion population to some extent thermalizes behind the obstacle, due to scattering by the fluctuations. The main feature seems to be that isotropic ion temperatures allow for wider excursions of structures in their motion in the direction perpendicular to the flow velocity. Larger obstacles tend to give rise to the formation of somewhat wider ("fatter") structures. For a diameter of $1.5 \lambda_{i}$ of the obstacle, it is no longer possible to observe any structures, but for a diameter of $2.5 \lambda_{i}$ or larger, they are clearly discernible. There is a significant difference between the dynamics of phase space vortices in one and two dimensions (Pécseli, 1987). The difference between a two-
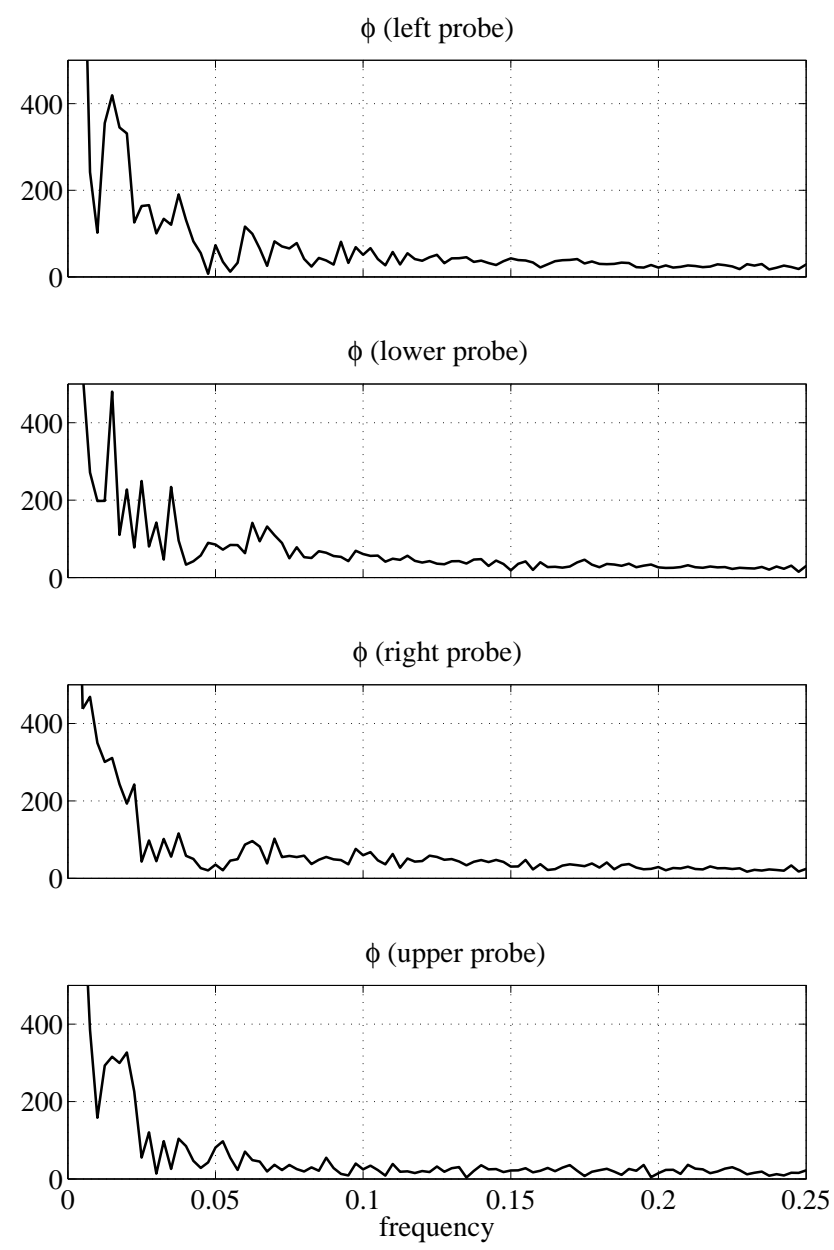

Fig. 10. Fluctuation amplitude spectra obtained for signals like those shown in Fig. 9, here with duration $400 / \Omega_{p i}$.

and three-dimensional simulation of a problem with cylindrical symmetry seems not to give significant differences on the time scales considered here.

\section{Discussions}

Long-lived potential structures have been observed in laboratory experiments, as well as numerical simulations. Also, many satellite observations from plasmas in the Earth's near environment have revealed the presence of seemingly longlived structures. Often the linear Landau damping is expected to be large in these conditions, and such structures should have a modest lifetime, if interpreted in the framework of a linear model. It is well known, however, that nonlinear wave phenomena can distort the velocity distribution locally, in such a way that the linear damping mechanisms become ineffective. Many numerical and experimental results have demonstrated that vortical structures in phase space have these characteristics. The properties of the individual structures is thus relatively well understood. Their 


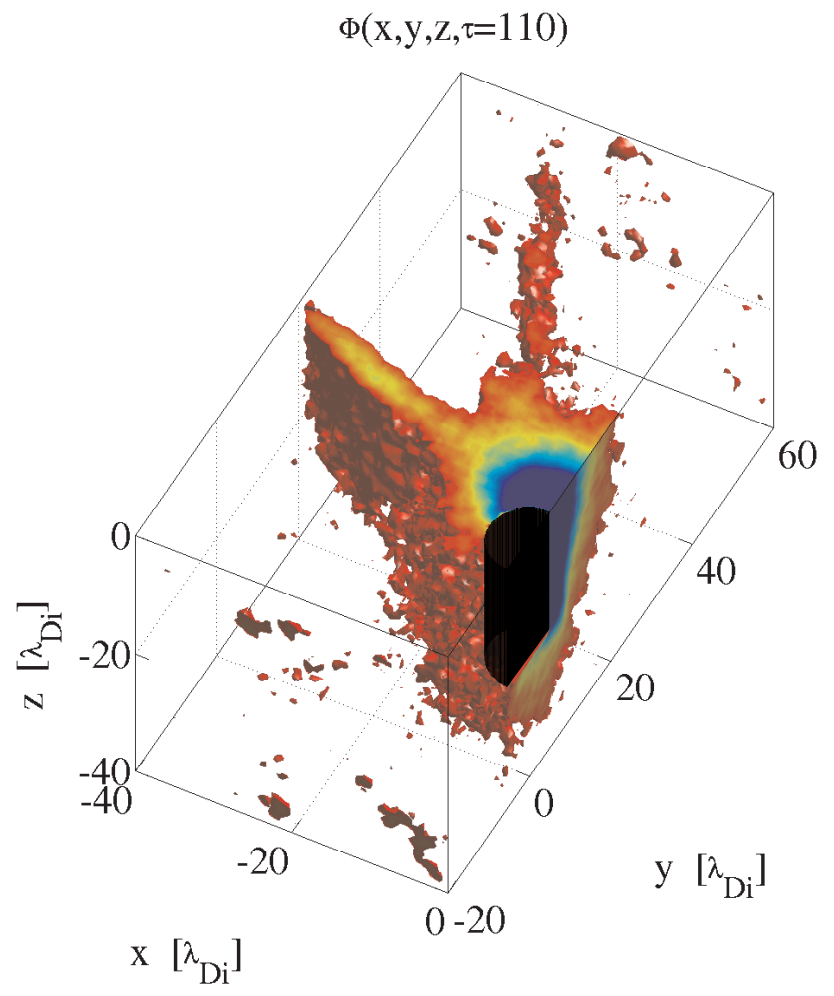

Fig. 11. Three-dimensional presentation of the potential variation around a cylinder segment placed in the ion flow. Only one-quarter of the simulation domain is shown. The corresponding quarter of the cylinder segment is shown in black color. Plasma parameters are $T_{i \|}=T_{i \perp}=T_{e} / 20$, and we have the plasma drift velocity to be $V_{0}=C_{S} / 2.05$.

formation mechanism is, however, still subject to controversy, and it might be that no unified model can be found.

In simulations with full electron, as well as ion dynamics (Omura et al., 2003), phase space structures were observed in the ion, as well as the electron phase space (best seen in electron phase space), as a result of an applied electric field which drives a current along magnetic field lines. Those results are expected to be relevant for the interpretation of data from the GEOTAIL spacecraft. These simulations indicate that the origin of the phase space structures is a kinetic current driven instability. Kinetic instabilities saturating in phase space vortices have been observed in simulations (in one spatial dimension) of electrostatic double-layers as well (Ergun et al., 2003), and the results related to observation in space plasmas. A combined analytical-numerical study of ion-ion beam instabilities by Pécseli and Trulsen (1982) demonstrated that also in this case ion phase space structures were formed as a result of a linear kinetic instability. In the present case, we have the initial local ion distribution being a drifting Maxwellian everywhere, and deviations from this develop due to the presence of the obstacle.

The plasma is unstable, due to a velocity shear instability. In this case we are dealing with a compressible flow, rather than the "classical" incompressible case (Chan-
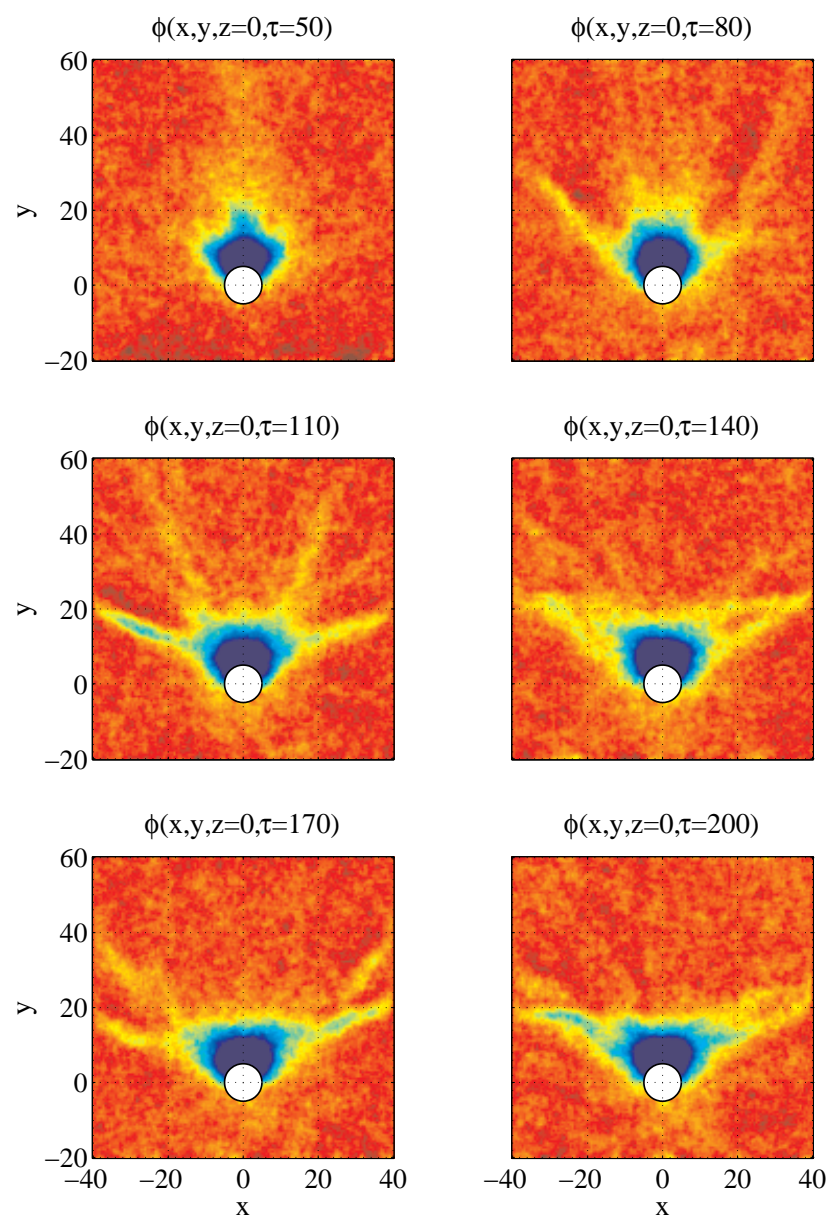

Fig. 12. Time evolution of the potential variation in the mid-plane of Fig. 11, shown for selected times.

drasekhar, 1961). In Fig. 13, we demonstrate that a localized velocity shear is present in the flow. The average or fluid velocity here is obtained numerically by integrating the velocity distribution to yield

$\mathbf{V}(\mathbf{r}, t) \equiv \frac{\iint \mathbf{v} f(\mathbf{r}, \mathbf{v}, t) d v_{x} d v_{y}}{\iint f(\mathbf{r}, \mathbf{v}, t) d v_{x} d v_{y}}$,

given by small arrows, while the color coding gives the potential variation as in the previous figures. As expected, the shear is found at the edge of the obstacle: in the shadow of the cylinder we have the plasma moving slowly, while it flows unimpeded past the object outside the shadow region. We see clearly a "channel" of small flow velocity behind the obstacle, with larger velocity vectors in the surrounding flow. The region of pronounced velocity shear is the one where the potential structures develop, (see Figs. 1) and 2. In presenting Fig. 13, we selected $t=70$, i.e. a time where the potential structures begin to develop (see Fig. 1). 


\subsection{Stability analysis}

Two possible but basically different instability mechanisms can be identified: one is a fluid type instability associated with the velocity shear that exists at the edges of the shadow region, and the other one is a kinetic instability generated by the oppositely directed ion beams that flow into the wake region from the two sides of the shadow region.

\subsection{Velocity shear}

To obtain a theoretical stability analysis for the velocity shear, we simplify the problem by considering a uniform shear $\mathbf{V}_{0}(\mathbf{r})=\left\{0, V_{0}(x), 0\right\}$. We ignore a density variation, since this will only give a modification of the linear growth rates, which will be approximate in the present model anyhow. In case we also allow for the density to be nonuniform, the unperturbed state will become complicated, since the electron pressure will give a dc-electric field in that case. This field will in turn affect the unperturbed velocity field, rendering it nonuniform.

We use the basic linearized momentum equation for a cold ion distribution, directly applicable for the case with $T_{e} \gg T_{i}$. With the linearized ion continuity equation, Poisson's equation with Boltzmann distributed electrons, we can Fourier transform with respect to the temporal variable, as well as the $z$-variable, along the unperturbed flow direction. As far as the $x$-direction is concerned we retain the full spatial variation.

We finally obtain one closed equation for the normalized potential $\psi \equiv e \phi / T_{e}$ as

$$
\begin{aligned}
(\omega- & \left.V_{0}(x) k_{\|}\right)\left(\psi\left(1+\left(k_{\|} \lambda_{D}\right)^{2}\right)-\lambda_{D}^{2} \frac{d^{2} \psi}{d x^{2}}\right) \\
& -C_{s}^{2} k_{\|}^{2} \frac{\psi}{\omega-V_{0}(x) k_{\|}}+C_{s}^{2} \frac{d \psi}{d x} \frac{d}{d x}\left(\frac{1}{\omega-V_{0}(x) k_{\|}}\right) \\
+ & C_{s}^{2} \frac{d}{d x}\left(\frac{\psi}{\omega-V_{0}(x) k_{\|}}\right)=0
\end{aligned}
$$

where we introduced the sound speed $C_{s} \equiv \sqrt{T_{e} / M}$. The relation can be seen, in general, as an complex eigen value problem for $\omega$ and corresponding eigen functions $\psi$, subject to the constraint that $\psi$ is finite for $x \rightarrow \pm \infty$.

Unfortunately, relation (2) is difficult to solve, even in the case where we assume the velocity shear to be a simple stepfunction. We consider here a localized solution, with the assumption of $V_{0} \approx \beta x$, which we analyze in the vicinity of $x=0$. By a change in the frame of reference, this approximation can be made valid. Since this assumed that the limiting case essentially corresponds to considering fine structures in the direction perpendicular to the shear velocity, we ignore terms containing the product $k_{\|} \lambda_{D}$. In this limit Eq. (2) simplifies to

$\frac{d^{2} \psi}{d x^{2}}+2 \beta \frac{k_{\|}}{\omega} \frac{d \psi}{d x}+\left(\frac{\omega^{2}}{C_{s}^{2}}-k_{\|}^{2}\right) \psi=0$.

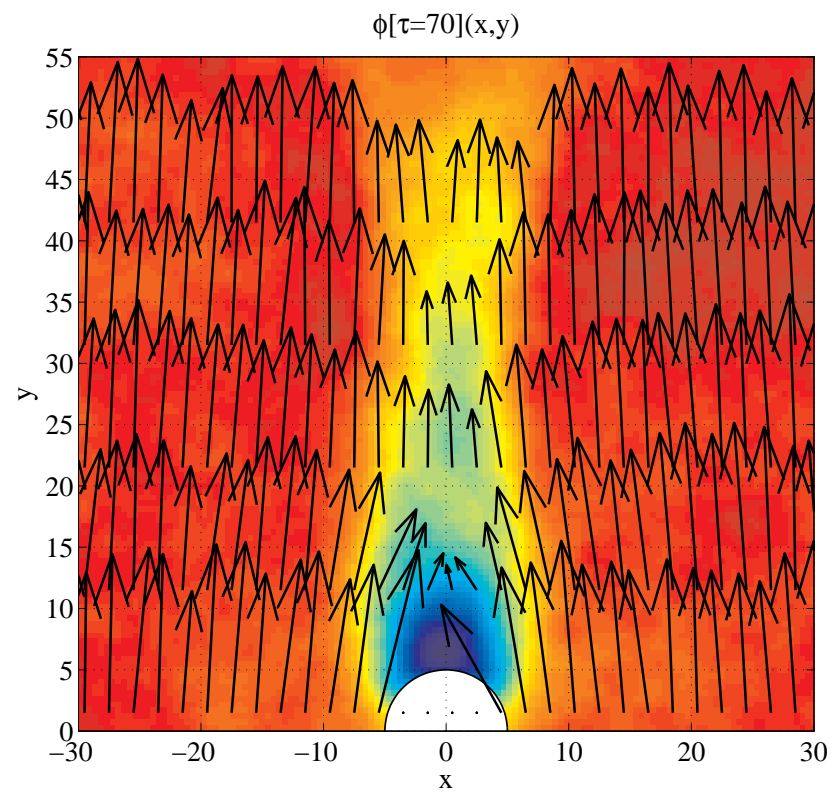

Fig. 13. Diagram for the plasma flow velocity. For simplicity, we show only a restricted domain of the simulation space, see Fig. 1 for color coding. The green-yellow transition is at $\phi \approx-T_{i} / e$.

The solution has the form $\psi=C_{1} \exp \left(\kappa_{1 \perp} x\right)+C_{2} \exp \left(\kappa_{2} \perp x\right)$, with $\kappa_{1} \perp$ and $\kappa_{2} \perp$ being solutions to the characteristic equation $\kappa_{\perp}^{2}+2 \beta \kappa_{\perp} k_{\|} / \omega+\left(\omega^{2} / C_{s}^{2}-k_{\|}^{2}\right)=0$.

In order for Eq. (3) to have bounded solutions satisfying the constraints at $x \rightarrow \pm \infty$ with $\kappa_{\perp, \|}$ real, we find that the frequency has to be purely imaginary, $\omega \equiv i \gamma$, corresponding to aperiodically growing solutions, in this particular frame of reference. In the limit where $\gamma$ is small, we readily find the approximate solution $\gamma=2 \beta k_{\perp} k_{\|} / k^{2}$, which implies $\kappa=i k_{\perp}$ in the solution for $\psi$, giving bounded solutions. More general results can be obtained by Euler's differential equation as a local approximation around $x=0$.

We note that a finite ion temperature within a fluid model will merely act to modify the sound speed slightly, and its inclusion is therefore of little consequence, apart from adding to the algebra.

\subsection{Kinetic instability}

The kinetic instability mentioned before is reminiscent of the one studied by, for instance, Singh and Schunk (1983); Singh et al. (1986, 1989); Samir et al. (1989), although those investigations were primarily concerned with objects moving at supersonic speeds. Basically, the beams enter from both sides of the shadow region, and when they mix, a two-stream region may develop. For the present condition, where the flow is sub-sonic, a nontrivial part of the tail population of the Maxwellian ion distribution can flow into the shadow region, and as far as the $\left\{x, v_{x}\right\}$-part ion phase space is concerned, see Fig. 3, it contributes with a particle population at zero average velocity. For a fixed Mach number of the flow (here with $\left.M \equiv V_{0} / C_{S}<1\right)$, the density of this component decreases 
with increasing temperature ratio $T_{e} / T_{i}$, which will imply $u_{t i} / C_{s}$ decreasing.

We can estimate the $x$-component of the ion beam velocity by noting that potential drops at the edges of the shadow region are at most $\Delta \phi \approx 0.2 T_{e} / e$, for the case analyzed here (see Fig. 13). Consequently, the $v_{x}$-component of the average ion velocity is of the order of the ion thermal velocity in the wake-region. In a homogeneous plasma, this velocity is insufficient to give rise to an ion-ion beam instability (Fried and Wong, 1966), but in the present shear flow it might be that the two instabilities enhance each other. We mention that in estimating the perpendicular ion temperature, $T_{i \perp}$, in the wake (or shadow) region, we must take into account the adiabatic cooling due to the acceleration of the ion component at the potential drop at the wake boundary. As mentioned before, counter-propagating ion beams can be seen in the wake region, e.g. Fig. 8, but at least in this particular case the ionbeam instability is unlikely to be operative, since vortex-like structures form at the high velocity sides of the beams, rather than between the two beams in phase space.

Our preference for the shear instability in the interpretation of the present simulations, Fig. 13, originates from the observation that the structures seem to develop at the location of the maximum shear, and at a direction almost normal to the gradient in plasma velocity. It is, however, evident that the relative importance of the two instabilities may change, depending on plasma parameters, the electron-ion temperature ratio in particular.

The phase space structures we observe thus represent the saturated stage of a linear instability. We expect the lifetime of the structures to be infinite, since they are constantly maintained in the wake behind the obstacle. Their length in the ion streamwise direction is, however, finite. There are not the same particles which constitute a structure at all times. Studies of the lifetime of ion phase space vortices (Pécseli et al., 1984; Børve et al., 2001) refer to initial value problems, and do not apply for the present case. If ion phase space vortices were to be analyzed as initial value problems with these plasma parameters, the resulting structures would appear as transient phenomena.

We emphasize that the omission of an externally imposed magnetic field is deliberate: it is known that ion phase space vortices (as well as their electron counterparts) have a lifetime which is increasing with the strength of the magnetic field (Børve et al., 2001). Our code allows for the inclusion of magnetic fields, but we choose the "worst-case" of an unmagnetized plasma, where the vortex lifetime is the smallest.

\section{Conclusions}

In the present communication, we studied plasma flows past an absorbing obstacle, with particular attention to subsonic flow velocities. We demonstrated that the wake behind the obstacle (see Fig. 13) is associated with a reduced plasma drift, as to be expected, while we have a larger flow velocity in the surrounding plasma. The resulting localized velocity shear is unstable, and breaks up into fine structures, which in the saturated stage turn out to be associated with phase space vortices (see Fig. 3). We find evidence for phase space coalescence of the phase space vortices, but this is in almost all cases a transient event, in contrast to other related studies, where coalesce is often an irreversible event (Saeki et al., 1979; Lynov et al., 1979, 1980). Here we are considering a boundary value problem, i.e. continuously maintained vortices, in contrast to most previous studies with initial value problems.

Phenomena interpreted as phase space structures are often observed by instrumented satellites (see reviews by, e.g. Guio et al. (2003)) or Krasovsky et al. (2003). Examples are found, for instance, in data also from the FREJA satellite. Sometimes such structures are accompanied by wave activity, e.g. lower-hybrid waves, but often not (Dovner et al., 1994; Pécseli et al., 1996). Care should be taken to distinguish the different properties of the observations. Often the structures are claimed to be uniquely associated with electron phase space structures, but we should bear in mind that the distinction between ion and electron phase space vortices is not evident if made on the basis of a density depletion and a double polarity of an electric field: in this case a signal from a spacecraft overtaking an ion hole cannot be discriminated from an electron hole overtaking the spacecraft. In both cases we observe a small density depletion associated with first a positive and then a negative electric field spike. Unambiguous interpretations are only possible if the phase space information is available, and this is rarely so, even in laboratory experiments (although the first observation of ion phase space vortices (Pécseli et al., 1981, 1984) was made in part by measuring the ion velocity distribution functions). In space observations, the evidence is mostly circumstantial.

One somewhat unpleasant observation based on our studies can be that obstacles in a plasma flow (or obstacles moving with moderate velocities through a stationary plasma) can generate phase space structures. If we associate an obstacle like the one investigated in the present study with a rocket or a satellite, or a part of one, we might anticipate that the longlived structures can be observed by probes on the same spacecraft, and incorrectly be associated with natural phenomena occurring in the ambient plasma. We see it as a problem that very often pulses attributed to phase space structures are seen in an environment where no formation mechanism is readily identified. On the other hand, such phase space structures have a lifetime which is large only in strongly magnetized plasmas, i.e. for $\omega_{c e} \geq \omega_{p e}$ for electron holes, and $\Omega_{c i} \geq \Omega_{p i}$ for their ion counterparts (Børve et al., 2001). These conditions are only sometimes fulfilled in space plasmas, but very easily in laboratory plasmas (Saeki et al., 1979; Lynov et al., 1979), and for a phase space vortex to be observed by a spacecraft, we expect it has to be close to the generation region. It is, however, only in some cases that a generation mechanism can be identified simultaneously with observations of phase space structures. The only obvious generation mechanism which is always present is the satellite itself! This scenario deserves, in our opinion, some attention. The 
signal representing the plasma phenomena can be "contaminated", so to speak, by structures generated by the spacecraft itself. This can happen in other cases as well: it is thus known (Cairns and Gurnett, 1991; Feng et al., 1993; Keller et al., 1997) that lower hybrid waves can be generated in the plasma density gradients of a magnetic field-aligned shadow region, behind a rapidly moving spacecraft. In this case these spurious signals are strictly confined to the wake, and will not be confused with lower hybrid waves naturally occurring in the plasma, as detected by, for instance, the FREJA satellite (Dovner et al., 1994; Pécseli et al., 1996), where lowerhybrid wave-filled cavities are detected with a probability independent of the satellite spin angle.

A high altitude satellite like the GEOTAIL spacecraft is small in comparison to the local electron Debye length (with $n \approx 0.1 \times 10^{6} \mathrm{~m}^{-3}, T_{e} \approx 100 \mathrm{eV}$ ). The FAST satellite is comparable to or larger than the Debye length (with $n \approx 6 \times 10^{6} \mathrm{~m}^{-3}$, $\left.T_{e} \approx 700 \mathrm{eV}\right)$. Therefore, these two satellites are close to being ineffective "point-sources", in the present context. However, a lower altitude satellite like FREJA is approximately $30 \times 6 \lambda_{D e}$, in terms of local plasma parameters. With a typical sound speed of $(7.4 \pm 15 \%) \times 10^{6} \mathrm{~m} \mathrm{~s}^{-1}$ (taken from standard tables) for a hydrogen plasma, and a satellite speed of $7.5 \times 10^{6} \mathrm{~m} \mathrm{~s}^{-1}$, we have the FREJA satellite propagating with Mach numbers varying around unity. Indeed, observations of sporadic localized structures have been reported (Dovner et al., 1994), in addition to wave-filled cavities, which are not discussed here. We also expect our results to apply for sounding rockets probing the collisionless plasma in the ionospheric E-region. It might also be mentioned that the instruments on board the low altitude Astrid2 satellite have detected significant satellite induced perturbations of the local plasma environment (Ivchenko et al., 2001), although the precise nature of these perturbations remains to be investigated. For the Astrid-2 satellite we have typical plasma parameters being densities in the range $8 \times 10^{3}-14 \times 10^{3} \mathrm{~cm}^{-3}$ and $T_{e} \approx 0.25 \mathrm{eV}$, giving $\lambda_{D e}$ in the range $3-4 \mathrm{~cm}$, which is much less than the geometrical size of the satellite.

We note that the solid object evidently need not be a part of a man-made spacecraft, it might as well represent a charged boulder propagating at a subsonic velocity. The problem might thus be applicable also to the dusty environment of planets like Saturn. One difference from the analysis in the present study can be that these "rocks" can be isolators, and that the electrostatic potential varies over the surface. The present study assumes constant potentials, as found for conducting objects, such as satellites or rockets.

The present problem contains, unfortunately, a very large number of parameters, and a complete coverage of the parameter space is not feasible. We have in terms of dimensionless numbers the parameters $V_{0} / C_{s}, e \Psi_{o} / T_{e}, T_{e} / T_{i}$, $T_{i \|} / T_{i \perp}$, and $D / \lambda_{D e}$, where $D$ is the diameter of the object, assumed to be cylindrical. In three-dimensional models we also have the parameter $D_{\|} / D_{\perp}$, allowing for elongated objects of finite lengths. If an externally imposed homogeneous magnetic field is included, we have in addition the param- eters $\Omega_{p i} / \Omega_{c i}, D \Omega_{c i} / C_{s}$, as well as the angle $\Theta$ between the velocity vector of the moving object and the magnetic field. In the present analysis we considered various values of $V_{0} / C_{s}, e \Psi_{o} / T_{e}$, and $T_{e} / T_{i}$. If collisional plasmas are considered, we have also the dimensionless parameter $v_{i} / \Omega_{p i}$, where $v_{i}$ is the ion collision frequency, with a neutral component, for instance, which would be relevant for the lower parts of the Earth's ionosphere. With the a priori assumption of Boltzmann distributed electrons, we need not consider an electron collision frequency. The effect of ion-neutral collisions on the ion vortex lifetime was studied by Pécseli et al. (1984), and it was found that the lifetime was reduced by collisions, giving a lifetime $\sim v_{i}^{-1}$, as expected. For the present case, with the vortices continuously maintained by the flow, we will observe a reduced length of the filaments behind the moving obstacle, as the collision frequency is increased.

The observations reported in the present study are expected to be relevant for supersonic conditions as well, although we emphasized here subsonic conditions, to avoid the influence of the Mach-cones. For more general conditions we have possibilities for considering irregular bounding surfaces, and probably more important, for velocity distribution functions deviating from Maxwellians. As far as the ion distributions are concerned, our numerical code poses no problems for including more general forms, but evidently, the electron distribution here will always be a Maxwellian, by construction of the code. The analysis presented in this paper is restrictive by ignoring magnetic fields, but as already mentioned, this is the "worst possible" case, since it was found (Børve et al., 2001) that it is in that case ion phase space vortices are most difficult to excite and maintain.

A number of future studies can be suggested: it would, for instance, be worthwhile to carry out a detailed analysis of the polarity of the electric fields of the observed bipolar structures in the rest frame of the plasma, which requires the plasma bulk flow velocity to be accurately determined along as well as across magnetic field lines. The correlation between the velocity of the observed structures and the electric field polarity (i.e. fields pointing towards or out of the density depletion, respectively) should be consistent with the velocity in the sense that an ion vortex should have velocities in the range $\left\{-u_{t i}, u_{t i}\right\}$, and electron vortices $\left\{-u_{t e}, u_{t e}\right\}$, in terms of the appropriate thermal velocities. It is true that formally we can envisage, for instance, ion vortices to have velocities noticeably larger than $u_{t i}$, but will emphasize that this requires rather "artificial" velocity distributions. The distribution of the positions of the observed structures with respect to the wake of the spacecraft (i.e. the spin phase) should be determined as well.

The analysis summarized in the present study was restricted to subsonic motion, in order to avoid influence of the Mach-cone studied previously by the same code (Guio and Pécseli, 2003). In particular, for magnetized plasmas, we expect this type of disturbance to be significant, since the radiation patterns can become very complicated in those cases (Trulsen and Fejer, 1970). The extension of our results to magnetized plasmas implies a significant data analysis. Our 
previous experience (Børve et al., 2001) indicates that the ion cyclotron frequency should be comparable to, or maybe even larger than, the ion plasma frequency, for the magnetic field to increase the vortex lifetime substantially.

Acknowledgement. This work was in part supported by the Norwegian Research Council's grant 146467/420, "Solar and Heliospheric Plasmas". The study was completed while one of the authors was associated with the Norwegian Centre for Advanced Study. The kind hospitality of this centre is gratefully acknowledged.

Topical Editor T. Pulkkinen thanks two referees for their help in evaluating this paper.

\section{References}

Bernstein, I. B., Greene, J. M., and Kruskal, M. D.: Exact nonlinear plasma oscillations, Phys. Rev., 108, 546-550, 1957.

Birch, P. C. and Chapman, S. C.: Particle-in-cell simulations of the lunar wake with high phase space resolution, Geophys. Res. Lett., 28, 219-222, 2001.

Birch, P. C. and Chapman, S. C.: Two dimensional particle-in-cell simulations of the lunar wake, Phys. Plasmas, 9, 1785-1789, 2002

Børve, S., Pécseli, H. L., and Trulsen, J.: Ion phase-space vortices in 2.5-dimensional simulations, J. Plasma Phys., 65, 107-129, 2001

Bujarbarua, S. and Schamel, H.: Theory of finite-amplitude electron and ion holes, J. Plasma Phys., 25, 515-529, 1981.

Cairns, I. H. and Gurnett, D. A.: Plasma-waves observed in the near vicinity of the space-shuttle, J. Geophys. Res., 96, 13913$13929,1991$.

Chandrasekhar, S.: Hydrodynamic and Hydromagnetic Stability, Oxford University Press, London, 1961.

Daldorff, L. K. S., Guio, P., Børve, S., Pécseli, H. L., and Trulsen, J.: Ion phase space vortices in 3 spatial dimensions, Europhys. Lett., 54, 161-167, 2001.

Dovner, P. O., Eriksson, A. I., Boström, R., and Holback, B.: Freja multiprobe observations of electrostatic solitary structures, Geophys. Res. Lett., 21, 1827-1830, 1994.

Ergun, R. E., Andersson, L., Carlson, C. W., Newman, D. L., and Goldman, M. V.: Double layers in the downward current region of the aurora, Nonlin. Processes Geophys., 10, 45-52, 2003.

Farrell, W. M., Kaiser, M. L., and Steinberg, J. T.: Electrostatic instability in the central lunar wake: a process for replenishing the plasma void?, Geophys. Res. Lett., 24, 1135-1138, 1997.

Farrell, W. M., Kaiser, M. L., and Steinberg, J. T.: A simple simulation of a plasma void: applications to Wind observations of the lunar wake, J. Geophys. Res., 103, 23 653-23 660, 1998.

Feng, W., Gurnett, D. A., and Cairns, I. H.: Interference patterns in the Spacelab-2 plasma-wave data - lower-hybrid waves driven by pickup, J. Geophys. Res., 98, 21 571-21 580, 1993.

Fried, B. D. and Wong, A. Y.: Stability limits for longitudinal waves in ion beam-plasma interaction, Phys. Fluids, 9, 10841089, 1966

Guio, P. and Pécseli, H. L.: Radiation of sound from a charged dust particle moving at high velocity, Plasma Phys., 10, 2667-2676, 2003.

Guio, P. and Pécseli, H. L.: Phase space structures generated by an absorbing obstacle in a streaming plasma, Geophys. Res. Lett., 31, doi:10.1029/2003GL018461, 2004.
Guio, P., Børve, S., Pécseli, H. L., and Trulsen, J.: Low frequency waves in plasmas with spatially varying electron temperature, Ann. Geophys., 18, 1613-1622, 2001.

Guio, P., Børve, S., Daldorff, L. K. S., Lynov, J. P., Michelsen, L. P., Pécseli, H. L., Rasmussen, J. J., Saeki, K., and Trulsen, J.: Phase space vortices in collisionless plasmas, Nonlin. Processes Geophys., 10, 75-86, 2003.

Hartquist, T. W., Havnes, O., and Morfill, G. E.: The effects of charged dust on Saturn's rings, Astronomy and Astrophys., 44, 26-30, 2003.

Ivchenko, N., Lindquist, P. A., Kekkonen, P., and Holback, B.: Disturbance of plasma environment in the vicinity of the Astrid-2 microsatellite, Ann. Geophys., 19, 655-666, 2001.

Ivlev, A. V., Khrapak, S. A., Zhdanov, S. K., Morfill, G. E., and Joyce, G.: Force on a charged test particle in a collisional flowing plasma, Phys. Rev. Lett., 92, 205007, 2004.

Jovanović, D. and Shukla, P. K.: Guiding center theory for ion holes in magnetized plasmas, Phys. Rev. Lett., 90, 135001, 2003.

Keller, A. E., Gurnett, D. A., Kurth, W. S., Yuan, Y., and Bhattacharjee, A.: Lower hybrid waves generated in the wake of the Galileo spacecraft, Planet. Space Sci., 45, 201-219, 1997.

Krasovsky, V. L., Matsumoto, H., and Omura, Y.: Electrostatic solitary waves as collective charges in a magnetospheric plasma: Physical structure and properties of BernesteinGreene-Kruskal (BGK) solitons, J. Geophys. Res., 108, doi:10.1029/2001JA000277, 2003.

Kwok, D. T.-K., Oates, T. W. H., McKenzie, D. R., and Bilck, M. M. M.: Determination of the equilibrium ion sheath in the drifting plasma by numerical simualtions, IEEE Trans. Plasma Sci., 31, 1044-1051, 2003.

Lynov, J. P., Michelsen, P., Pécseli, H. L., Rasmussen, J. J., Saeki, K., and Turikov, V.: Observations of solitary structures in a magnetized, plasma loaded waveguide, Phys. Scripta, 20, 328-335, 1979.

Lynov, J. P., Michelsen, P., Pécseli, H. L., and Rasmussen, J. J.: Interaction between electron holes in a strongly magnetized plasma, Phys. Lett., 80A, 23-25, 1980.

Newman, D. L., Goldman, M. V., Spector, M., and Perez, F.: Dynamics and instability of electron phase-space tubes, Phys. Rev. Lett., 86, 1239-1242, 2001.

Omura, Y., Heikkila, W. J., Umeda, T., Ninomiya, K., and Matsumoto, H.: Particle simulations of plasma response to an applied electric field parallel to magnetic field lines, J. Geophys. Res., 108, doi:10.1029/2002JA009573, 2003.

Oppenheim, M., Vetoulis, G., Newman, D. L., and Goldman, M. V.: Evolution of electron phase-space holes in 3-D, Geophys. Res. Lett., 28, 1891-1894, 2001.

Pal, A. R., Boruah, D., Bailung, H., and Chutia, J.: Influence of low energy ion beam on sheath characteristics in a plasma, Phys. Lett. A, 305, 419-426, 2002.

Pécseli, H. L.: Ion phase-space vortices and their relation to small amplitude double layers, Laser Part. Beams, 5, 211-217, 1987.

Pécseli, H. L. and Trulsen, J.: Nonlinear evolution of the ion-ion beam instability, Phys. Rev. Lett., 48, 1355-1358, 1982.

Pécseli, H. L., Trulsen, J., and Armstrong, R.: Experimental observation of ion phase-space vortices, Phys. Lett., 81A, 386-390, 1981.

Pécseli, H. L., Trulsen, J., and Armstrong, R.: Formation of ion phase-space vortexes, Phys. Scripta, 29, 241-253, 1984.

Pécseli, H. L., Iranpour, K., Holter, Ø., Lybekk, B., Holtet, J., Trulsen, J., Eriksson, A., and Holback, B.: Lower hybrid wave cavities detected by the FREJA satellite, J. Geophys. Res., 101, 
5299-5316, 1996.

Saeki, K., Michelsen, P., Pécseli, H. L., and Rasmussen, J. J.: Formation and coalescence of electron solitary holes, Phys. Rev. Lett., 42, 501-504, 1979.

Sakanaka, P. H.: Beam-generated collisionless ion-acoustic shocks, Phys. Fluids, 15, 1323-1327, 1972.

Samir, U., Comfort, R. H., Singh, N., Hwang, K. S., and Stone, N. H.: Insight into theory-experiment comparisons of wake measurements in the plasmasphere, Planet. Space. Sci., 37, 873-880, 1989.

Schamel, H. and Bujarbarua, S.: Solitary plasma hole via ion-vortex distribution, Phys. Fluids, 23, 2498-2499, 1980.

Shukla, P. K.: Dust Plasma Interaction in Space, Nove Science, 2002.

Singh, N. and Schunk, R. W.: Numerical simulations of counterstreaming plasmas and their relevance to the interhemispheric flows, J. Geophys. Res., 88, 7867-7877, 1983.

Singh, N., Thiemann, H., and Schunk, R. W.: Studies of counterstreaming plasma expansion, Phys. Scripta, 33, 355-369, 1986.
Singh, N., Wright Jr, K. H., Stone, N. H., Samir, U., and Hwang, K. S.: On the interpretation of measured ion streams in the wake of the shuttle orbiter in terms of plasma expansion processes, J. Geophys. Res., 94, 12 075-12 080, 1989.

Singh, N., Loo, S. M., and Wells, E.: Electron hole as an antenna radiating plasma waves, Geophys. Res. Lett., 28, 1371-1374, 2001.

Stangeby, P. C. and Allen, J. E.: Plasma boundary as a Mach surface, J. Phys. A: Gen. Phys., 6, 304-308, 1970.

Stangeby, P. C. and Allen, J. E.: Transonic plasma flow past an obstacle, J. Plasma Phys., 6, 19-32, 1971.

Trulsen, J. and Fejer, J. A.: Radiation from a charged particle in a magnetoplasma, J. Plasma Phys., 4, 825-841, 1970.

Tskhakaya, D., Shukla, P., and Eliasson, B.: Potential around a dust grain placed in the plasma sheath, Phys. Lett., A331, 405-408, 2004.

Wesseling, P.: An Introduction to Multigrid Methods, John Wiley and Sons Ltd, 1991. 